\title{
The Role of Oxidative Damage in the Pathogenesis and Progression of Alzheimer's Disease and Vascular Dementia
}

\author{
Maria Luca, ${ }^{1}$ Antonina Luca, ${ }^{2}$ and Carmela Calandra ${ }^{1}$ \\ ${ }^{1}$ Psychiatry Unit, Department of Medical and Surgical Sciences and Advanced Technologies, \\ University Hospital Policlinico-Vittorio Emanuele, Santa Sofia Street 78, Catania, 95100 Sicily, Italy \\ ${ }^{2}$ Department of "G.F. Ingrassia", University Hospital Policlinico-Vittorio Emanuele, Santa Sofia Street 78, Catania, \\ 95100 Sicily, Italy \\ Correspondence should be addressed to Carmela Calandra; c.calandra@unict.it
}

Received 5 February 2015; Accepted 8 April 2015

Academic Editor: Verónica Pérez de la Cruz

Copyright ( $\odot 2015$ Maria Luca et al. This is an open access article distributed under the Creative Commons Attribution License, which permits unrestricted use, distribution, and reproduction in any medium, provided the original work is properly cited.

Oxidative stress (OS) has been demonstrated to be involved in the pathogenesis of the two major types of dementia: Alzheimer's disease $(\mathrm{AD})$ and vascular dementia $(\mathrm{VaD})$. Evidence of $\mathrm{OS}$ and OS-related damage in $\mathrm{AD}$ is largely reported in the literature. Moreover, OS is not only linked to $\mathrm{VaD}$, but also to all its risk factors. Several researches have been conducted in order to investigate whether antioxidant therapy exerts a role in the prevention and treatment of $\mathrm{AD}$ and $\mathrm{VaD}$. Another research field is that pertaining to the heat shock proteins $\left(\mathrm{Hsp}_{\mathrm{s}}\right)$, that has provided promising findings. However, the role of OS antioxidant defence system and more generally stress responses is very complex. Hence, research on this topic should be improved in order to reach further knowledge and discover new therapeutic strategies to face a disorder with such a high burden which is dementia.

\section{Oxidative Stress and Brain Aging}

Redox homeostasis is a complex mechanism that can be resumed as the maintenance of the balance between reactive oxygen species (ROS) production and elimination [1]. Largely generated from mitochondria, ROS are by-products of cellular metabolism [2]. Among them, we include free radicals (superoxide), hydroxyl radicals (the most reactive species), and nonradicals (hydrogen peroxide). Even though ROS exert a role in crucial physiological processes, such as signaling and apoptosis $[3,4]$, they are highly reactive species; as a result, they can damage proteins, lipids, deoxyribonucleic acid (DNA), and sugars with remarkable negative consequences on the cellular functioning [5]. The antioxidant defence system, composed by nonenzymatic and enzymatic antioxidants (e.g., glutathione, flavonoids, superoxide dismutase (SOD), catalases, and glutathione peroxidase (GPx)), protects the cells from the ROS-related injuries [6]. If the redox homeostasis fails, this system is not sufficient to counteract the high amount of ROS and the so-called "oxidative stress" (OS) occurs [7]. The balance between oxidants and antioxidants is not a static condition and a great number of stimuli can interfere with the redox status. Hence, OS has been recently redefined as a "disruption of redox signaling and control" [8]. OS and its detrimental effects on the cellular functioning have been demonstrated to be involved in aging [9], as well as in a variety of illnesses, particularly age-related ones, among which are diabetes [10], atherosclerosis [11], mild cognitive impairment (MCI) [12], Parkinson's disease [13], and other neurodegenerative disorders, such as Huntington's disease [14] and amyotrophic lateral sclerosis [15]. In addition, OS seems to be involved in the pathogenesis of the two major types of dementia: Alzheimer's disease (AD) and vascular dementia ( $\mathrm{VaD})$ [16]. The importance of OS in so many neurodegenerative disorders is not surprising, since the brain is highly susceptible to ROS, because

(1) it is rich in fatty acids, which are sensible to peroxidation;

(2) it has not a powerful antioxidant activity;

(3) it consumes a lot of oxygen; therefore, it is exposed to free-radicals accumulation [17]. 
Previous researches highlighted the importance of OS in both normal brain aging and pathological brain aging [18], sharing the same altered biochemical and anatomopathological pattern: neural loss and altered mitochondrial activity and accumulation of degradated mitochondria and tangles [19-21]. However, AD brains show substantial qualitative and quantitative differences when compared to controls. More specifically, the redox homeostasis is different: the activity of mitochondrial pyruvate dehydrogenase, ketoglutarate dehydrogenase, and cytochrome oxidase is more severely affected; moreover, the antioxidant defence system is critically impaired $[20,22]$. In addition, even though normal brain aging is related to the accumulation of degradated mitochondria and tangles, both conditions are more represented in $\mathrm{AD}[20,21]$. The amount of tangles certainly increases with age, particularly in the hippocampus. However, it is remarkably higher in demented brains and the more the dementia is severe, the more the tangles affect the neocortex, which is usually spared in normal brain aging [21].

High levels of peripheral markers of oxidative stress and low antioxidant power have been reported in patients with $\mathrm{MCI}$, late onset $\mathrm{AD}$, and $\mathrm{VaD}$. Even though the three disorders seem to share a common oxidative-related pathogenesis, they maintain distinctive features, since other variables (e.g., homocysteine levels) allow for their differentiation [16]. An interesting study published in 2013 reported how young healthy individuals at risk of developing AD (as determined through genetic analysis) presented an apparently paradoxical condition: high levels of antioxidants and reductive, rather than oxidative, stress. On the contrary, in case of overt $\mathrm{AD}$, the opposite situation was noticed: low antioxidants and high indicators of oxidative stress. The hypothesis is that the individuals at risk of developing $\mathrm{AD}$, presenting an increased generation of ROS, respond with an overexpression of antioxidants, thus suffering from reductive stress. Later on, the antioxidant defence system collapses and the OS becomes evident, along with the symptoms of dementia [23]. The role of OS in the physiopathology of dementia is very complex and the knowledge pertaining to this topic needs to be enhanced. In the present review, data on the role of $\mathrm{OS}$ in $\mathrm{AD}$ and $\mathrm{VaD}$, as well as a discussion on the therapeutic implications of such a role, are reported.

\section{OS and AD}

In the last decades, several researches investigating the role of OS in neurodegenerative disorders have been conducted. Despite the fact that the knowledge on this topic is certainly larger than before, it is still unclear whether OS is the cause or the consequence of the neurodegenerative processes. Notwithstanding, it is almost certain that OS is involved in the crucial events leading to the neural death and in the propagation of such events. Hence, if the complex and various neurodegenerative phenomena are intended as a cycle for more than a cascade, it is clear that, one way or another, OS is the "main actor" [24]. AD, the most common cause of dementia in the elderly, is an age-related neurodegenerative disorder causing the progressive loss of the higher cerebral functions, such as memory, language, and cognitive thinking, with huge consequences on mood, behaviour, and self-sufficiency [25]. This type of dementia is characterized by the accumulation of misfolded beta-amyloid $(\mathrm{A} \beta)$, a protein produced from the cleavage of the amyloid precursor protein (APP), and neurofibrillary tangles (aggregates of hyperphosphorylated tau protein) in the brain $[26,27]$. Evidence of OS and OSrelated damage in $\mathrm{AD}$ is largely reported in the literature [27-30]. More specifically, markers of lipid peroxidation have been found in plasma, urine, and cerebral tissue of transgenic mice models of AD amyloidosis [31]; OS-related DNA damage has been demonstrated in $\mathrm{AD}$ patients, so that some authors suggested that urinary oxidized nucleosides could be used as biomarkers [32]; high levels of carbonyls, markers of protein oxidation, have been found in $\mathrm{AD}$ brains [33]. Also tau phosphorylation has been related to OS and it is known that hyperphosphorylation is responsible for its misfolding $[34,35]$. It is known that the ROS production is crucially involved in the physiological mechanisms regulating folding, misfolding, and the elimination of unfolded proteins [3638]. The endoplasmic reticulum (ER) plays a fundamental role in the regulation of protein folding. In case of abundance of misfolded proteins, ER stress occurs, thus determining an enhanced production of ROS during the oxidative folding process (formation of disulfide bonds) and the uncontrolled accumulation of unfolded/misfolded proteins [36, 39]. The consequential depletion of the antioxidant glutathione and the ROS-related damage of the mitochondrial electron transfer system amplify the production of ROS and lead to cell death. In fact, the ER alteration is one of the common features linking neurodegenerative disorders, mostly characterized by protein misfolding, to each other [36]. In addition, a sustained OS alters the functions of the ubiquitin-proteasome pathway, which is responsible for the degradation of the damaged proteins [38]. As far as $\mathrm{AD}$ is concerned, there are several conditions causing the excessive production of ROS: mitochondrial dysfunctions, $\mathrm{A} \beta$-related microglial activation, inflammation, and binding of redox active metals to deposits [40]. A deficiency in cytochrome c oxidase has been reported in platelets and in postmortem brain tissue of $\mathrm{AD}$ patients. As a result, mitochondrial degradation is stimulated and neurons are damaged by the mitochondrial debris and the excessive production of ROS [41]. As if that were not enough, the altered mitochondria generate high levels of ROS, exposing themselves to the OS-related injuries to which they are so sensitive [42]. Another important issue in AD is inflammation. In fact, this neurodegenerative disorder is characterized by an uncontrolled inflammatory activation of microglial cells [43]. The peroxisome proliferator-activated receptor gamma (PPAR- $\gamma$ ) is a regulator of the inflammatory processes which exerts anti-inflammatory properties [44]. OS, leading to the phosphorylation of PPAR $-\gamma$, is responsible for the functional alteration of this important transcription factor [45]. In addition, it has been reported that mild OS could trigger the amyloid cascade being, therefore, involved in the very early stages of AD: in fact, it causes an alteration of the subcellular compartmentalization of BACE1 (betasite APP cleaving enzyme 1), an enzyme involved in the $\beta$ secretase cleavage of the APP; as a result, the amyloidogenic processing of APP is favoured [46]. As in a vicious cycle, 
$\mathrm{A} \beta$ produces ROS through a metal-catalyzed reaction [47]. The lesions observed in brains suffering from $\mathrm{AD}$ are those typical of OS (e.g., damage to DNA, protein oxidation, and lipid peroxidation) [18] and contain metals (e.g., zinc, iron) exerting catalytic activity and causing ROS production [48]; these metals have been demonstrated to be highly represented in AD brains [49]. From what has been discussed above, it is apparent that OS is involved in the occurrence of the core aspects of $\mathrm{AD}$, that is, phosphorylation and misfolding [46, 50]. Obviously, the exposure of OS stimulates the activation of compensatory responses [51]. Unfortunately, both enzymatic and nonenzymatic antioxidant defences seem to be impaired in $\mathrm{AD}$ patients $[52,53]$. Even though literature data are not exempted from inconsistencies [22], it is plausible that SOD could be induced by $\mathrm{OS}$ in the early stages of $\mathrm{AD}$ and, then, consumed in the advanced stages [22]. GPx and glutathione reductase (GR) seem to be, respectively, higher and lower in $\mathrm{AD}$ patients versus controls. Hence, the GR/GPx activity ratio turns out to be higher in healthy subjects, intermediate in $\mathrm{MCI}$, and lower in $\mathrm{AD}$ patients. In addition, the ratio has been found to be positively correlated with the scores at the Mini Mental Sate Examination (MMSE), a tool used to assess the cognitive performances [12]. It is easy to imagine that since GR (which regenerates reduced glutathione from oxidized glutathione (GSSG)) is low, the levels of GSSG should be high. In fact, the levels of GSSG are higher in AD patients and relate to the severity of the dementia [22]. As far as nonenzymatic defences are concerned, even in this case literature data show some inconsistencies, but various studies report vitamin deficiency in patients versus controls [22]. Since the redox homeostasis is so deeply altered, the neurons are dangerously exposed to the detrimental effects of OS and to the fearsome mechanism of neurodegeneration.

\section{OS and VaD}

$\mathrm{VaD}$ is the second cause of dementia in the elderly. Executive functions, more than memory, are severely impaired in this type of dementia [54]. Even if hypoxia and haemorrhagic stroke (e.g., subdural haematoma) can cause $\mathrm{VaD}$, the latter is mostly related to ischaemic stroke [55]. In particular, one of the most common forms of $\mathrm{VaD}$, the subcortical one, is caused by multiple subcortical ischaemic lesions. Hypertension, diabetes mellitus, hypercholesterolemia, and hyperhomocysteinemia favour the occurrence of atherosclerosis, cardiovascular diseases, and stroke and represent important risk factors for $\mathrm{VaD}$ [54]. As far as oxidative stress is concerned, it is linked not only to $\mathrm{VaD}$, but also to all its risk factors; in fact, OS has been demonstrated to play a role in the pathogenesis of diabetes [10] and to be involved in the tissue toxicity determined by hypercholesterolemia [56] and hyperhomocysteinemia [57]. Moreover, it has been reported that the dysfunction of mitochondrial proteins, leading to OS, is involved in the hypertension-related target organ damage affecting vasculature, heart, kidneys, and brain [58]. Mitochondrial dysfunction is considered to be an important step in the pathogenesis of atherosclerosis, also because it subtends the previously mentioned risk factors [59]. The OSrelated oxidation of low-density lipoproteins (LDL) is crucial in the atherosclerotic process $[60,61]$ and high levels of lipid hydroperoxides have been reported in patients with ischemic stroke [62]. As a matter of fact, patients with $\mathrm{VaD}$ have been found to show high levels of malondialdehyde (a marker of lipid peroxidation) and these levels were higher than those reported in $\mathrm{AD}$ patients [63]. The association between folate deficiency and OS-related LDL dysfunction seems to be typical of $\mathrm{VaD}$ and could help to differentiate it from other types of dementia [64]. In addition, OS is certainly a mediator of the stroke-related neuronal damage and cognitive dysfunctions, as demonstrated by the high levels of plasmatic ROS in patients with ischaemic stroke as well as the finding of oxidative DNA damage within the peri-infarct brain regions in rats [65-67]. Moreover, OS is indirectly and directly involved in the deep alterations of the blood-brain barrier (BBB) occurring after an ischaemic brain injury. More specifically, the activation of metalloproteinases and the proteases involved in the proteolytic disruption of the $\mathrm{BBB}$ and in the white matter lesions typical of $\mathrm{VaD}$ is strictly linked to OS $[68,69]$. In addition, ROS are directly responsible for the alterations in the cerebral perfusion and permeability, thus contributing to the cerebrovascular disease [70]. The OS-induced dysfunction of the previously cited anti-inflammatory agent PPAR- $\gamma$ is involved in vascular aging [71]. In practice, OS and inflammation "cooperate" in determining the endothelial damage and the BBB failure occurring in $\mathrm{VaD}$ [72]. As in $\mathrm{AD}$, even in $\mathrm{VaD}$ the antioxidant defence system seems to be insufficient. Apart from the previously reported folate deficiency [64], also vitamin E has been found to be lower in $\mathrm{VaD}$ versus controls and also versus $\mathrm{AD}$ [73]. In addition, SOD and GR are reduced in $\mathrm{VaD}$ too [74]. However, it is worth pondering over that, from a clinical point of view, the difference between $\mathrm{AD}$ and $\mathrm{VaD}$ is not so strict. In fact, microvascular degeneration and atherosclerotic processes are often documented in $\mathrm{AD}$, in which an ROS-related amyloid cerebral angiopathy occurs [75-77]. In addition, the LDL oxidation, involved in the atherosclerotic process as previously mentioned $[60,61]$, seems to be a common feature shared by $\mathrm{AD}$ and $\mathrm{VaD}$ [78]. An important antioxidant enzymatic system, influenced by the redox status [79], protecting LDL from oxidation and exerting anti-inflammatory properties, is represented by the serum paraoxonases $[78,80]$. Paraoxonase activity (as a protective factor) and macrophage OS (as a deleterious condition) take part in atherogenesis [81, 82]. Additionally, macrophage OS has been related to the paraoxonase 1 deficiency [83]. Considering dementia, both $\mathrm{AD}$ and $\mathrm{VaD}$ patients have been found to show a lower paraoxonase activity when compared to controls [78]. In the light of what has been discussed, OS represents a common important contributor in the pathogenesis of these two forms of dementia, whether alone or in comorbidity (mixed $\mathrm{AD}$ and $\mathrm{VaD}$ ) [76]. Moreover, not only GR/GPx ratio, but also other markers of OS have been related to cognitive performances in dementia. High levels of 4-hydroxynonenal and malonaldehyde relate to worse scores at the MMSE [12, 84]. Notwithstanding, the use of biomarkers as predictors of 
severity or outcome of dementia seems to be not strongly enough supported, at least for now [22].

\section{Antioxidants in AD and VaD: Future Therapeutic Perspectives}

Several researches have been conducted in order to investigate whether antioxidant therapy exerts a role in the prevention and treatment of $\mathrm{AD}$ and $\mathrm{VaD}$. The findings arisen from a cohort study considering more than 1300 subjects indicate that the intake of flavonoids is inversely related to the risk of incident dementia [82]. Hence, vitamin supplementation could play a positive effect in both $\mathrm{AD}$ and $\mathrm{VaD}[85,86]$. It has been demonstrated that curcumin reduces the levels of $\mathrm{A} \beta$ in cell lines and mouse primary cortical neurons and exerts a neuroprotective effect in vascular dementia enhancing the expression of antioxidants in rats and ischemic cells [87, 88]. In fact, the enhancement of the enzymatic defences is the key of the efficacy of treatments such as the EUK-207 (SOD/catalase mimetic) in mice with $\mathrm{AD}$ and resveratrol in rats with $\mathrm{VaD}[89,90]$. It has been reported that resveratrol increases SOD activity and glutathione levels in the cerebral cortex and hippocampus of rats with $\mathrm{VaD}$ [90]. Another research field is pertaining to the heat shock proteins $\left(\mathrm{Hsp}_{\mathrm{s}}\right)$, which has provided interesting findings. It is well known that OS is involved in the activation of such important chaperons, which regulate the aggregation of misfolded proteins and apoptosis [91]. However, in case of neurodegeneration, the chaperones could paradoxically facilitate the aggregation of disease-related proteins, attempting to repair them and trying to avoid the formation of toxic aggregates [92]. This data explains why Hsp90 inhibitors are one of the promising therapeutic tools for the treatment of $\mathrm{AD}[93,94]$. It has been affirmed that Hsp90 "may play a role in maintaining pathogenic changes that lead to neurodegenerative diseases" [95]. This concept is easier to understand if it is considered that the tau protein is a client protein for Hsp90 [96] and that the latter is a repressor of the heat shock factor-1 (HSF-1), which regulates the heat shock response through the expression of $\mathrm{Hsp}_{\mathrm{s}}[93,97]$. Hsp90 inhibitors, through the activation of HSF-1, exert neuroprotective effects favouring the induction of $\mathrm{Hsp}_{\mathrm{s}}$, such as Hsp70 $[93,98]$, that has been found to exert therapeutic properties in mice with AD [99]. $\mathrm{Hsp}_{\mathrm{s}}$ are also expressed in response to ischemic brain lesions $[100,101]$ and transgenic mice expressing human inducible Hsp70 have shown to have ischemia-resistant hippocampal neurons [102]. Since inflammation plays an important role in the pathogenesis of $\mathrm{AD}$ and $\mathrm{VaD}[43,72]$, the enhancement of the anti-inflammatory defence, through PPAR- $\gamma$ agonists, could represent another potential target for the treatment of these severe dementias. In mice models of AD, PPAR$\gamma$ agonism resulted in the reduction of parenchymal $\mathrm{A} \beta$, microglial activation, and neural loss [43]. In addition, it showed efficacy in improving reversal learning [103]. The oral antidiabetic drugs pioglitazone and rosiglitazone, exerting agonistic properties on PPAR- $\gamma$, were found to reverse some clinical (memory, learning) and biochemical (OS, endothelial dysfunction) features of diabetes-induced $\mathrm{VaD}$ dementia
[104]. Moreover, their antiatherogenic effect is not only linked to their insulin sensitizing properties, but also to the modulation of endothelial activation markers, platelet activity, and vasodilatation. Hence, their therapeutic effects could be useful also for patients without diabetes mellitus affected by dementia [105]. However, the antioxidant therapy has not reached the aimed results. Among the more credible causes of this failure, (a) the activity of many nutritional antioxidants is strictly linked to that of other antioxidants; hence, monotherapy could not be sufficient; (b) therapy is often administered in too advanced stages of dementia; (c) since the brain is separated from the periphery trough the $\mathrm{BBB}$, the peripheral redox status may not reflect the cerebral homeostasis; (d) the researches available used different methods to analyze the antioxidant levels in blood in order to evaluate the outcome after therapy [106]. Therefore, there are many fascinating plausible therapeutic targets that need to be further investigated to add new and more efficient therapeutic tools to the nowadays available disappointing options.

\section{Concluding Remarks}

In the light of what has been discussed, OS seems to be a crucial contributor in the pathogenesis of $\mathrm{AD}$ and $\mathrm{VaD}$, directly or indirectly affecting the steps leading to neurodegeneration. $\mathrm{AD}$ and $\mathrm{VaD}$ are linked by many anatomical features, as well as by OS. However, the role of OS, antioxidant defence system and, more generally, stress responses, is very complex. Hence, research pertaining to this topic should be improved in order to reach further knowledge and discover new therapeutic strategies to face a disorder with such a high burden which is dementia.

\section{Conflict of Interests}

The authors declare that there is no conflict of interests regarding the publication of this paper.

\section{Authors' Contribution}

Maria Luca and Antonina Luca contributed equally to this work.

\section{References}

[1] M. Fiedorowicz and P. Grieb, "Nitrooxidative stress and neurodegeneration," in Brain Damage-Bridging Between Basic Research and Clinics, A. Gonzalez-Quevedo, Ed., chapter 6, InTech, Rijeka, Croatia, 2012.

[2] V. J. Thannickal and B. L. Fanburg, "Reactive oxygen species in cell signaling," The American Journal of Physiology-Lung Cellular and Molecular Physiology, vol. 279, no. 6, pp. L1005L1028, 2000.

[3] S. Bolisetty and E. A. Jaimes, "Mitochondria and reactive oxygen species: physiology and pathophysiology," International Journal of Molecular Sciences, vol. 14, no. 3, pp. 6306-6344, 2013.

[4] C. P. Baran, M. M. Zeigler, S. Tridandapani, and C. B. Marsh, "The role of ROS and RNS in regulating life and death of blood monocytes," Current Pharmaceutical Design, vol. 10, no. 8, pp. 855-866, 2004. 
[5] K. Ogino and D.-H. Wang, "Biomarkers of oxidative/nitrosative stress: an approach to disease prevention," Acta Medica Okayama, vol. 61, no. 4, pp. 181-189, 2007.

[6] H. Klandorf and K. van Dyke, "Oxidative and nitrosative stresses: their role in health and disease in man and birds," in Oxidative Stress-Molecular Mechanisms and Biological Effects, V. Lushchak and H. M. Semchyshyn, Eds., chapter 3, InTech, Rijeka, Croatia, 2012.

[7] H. Sies, "Oxidative stress: oxidants and antioxidants," Experimental Physiology, vol. 82, no. 2, pp. 291-295, 1997.

[8] D. P. Jones, "Redefining oxidative stress," Antioxidants and Redox Signaling, vol. 8, no. 9-10, pp. 1865-1879, 2006.

[9] P. Ljubuncic, E. Gochman, and A. Z. Reznick, "Nitrosative stress in aging-its importance and biological implications in NF-kB signaling," in Aging and Age-Related Disorders, S. Bondy and K. Maiese, Eds., vol. 3 of Oxidative Stress in Applied Basic Research and Clinical Practice, Springer, 2010.

[10] A. C. Maritim, R. A. Sanders, and J. B. Watkins III, "Diabetes, oxidative stress, and antioxidants: a review," Journal of Biochemical and Molecular Toxicology, vol. 17, no. 1, pp. 24-38, 2003.

[11] H. Li, S. Horke, and U. Förstermann, "Vascular oxidative stress, nitric oxide and atherosclerosis," Atherosclerosis, vol. 237, no. 1, pp. 208-219, 2014.

[12] L. L. Torres, N. B. Quaglio, G. T. de Souza et al., "Peripheral oxidative stress biomarkers in mild cognitive impairment and alzheimer's disease," Journal of Alzheimer's Disease, vol. 26, no. 1, pp. 59-68, 2011.

[13] A. Gaspar, N. Milhazes, L. Santana, E. Uriarte, F. Borges, and M. J. Matos, "Oxidative stress and neurodegenerative diseases: looking for a therapeutic solution inspired on benzopyran chemistry," Current Topics in Medicinal Chemistry, vol. 15, no. 5, pp. 432-445, 2015.

[14] S. E. Browne, R. J. Ferrante, and M. F. Beal, "Oxidative stress in Huntington's disease," Brain Pathology, vol. 9, no. 1, pp. 147-163, 1999.

[15] E. Radi, P. Formichi, C. Battisti, and A. Federico, "Apoptosis and oxidative stress in neurodegenerative diseases," Journal of Alzheimer's Disease, vol. 42, supplement 3, pp. S125-S152, 2014.

[16] C. Cervellati, A. Romani, D. Seripa et al., "Oxidative balance, homocysteine, and uric acid levels in older patients with Late Onset Alzheimer's Disease or Vascular Dementia," Journal of the Neurological Sciences, vol. 337, no. 1-2, pp. 156-161, 2014.

[17] B. Uttara, A. V. Singh, P. Zamboni, and R. T. Mahajan, "Oxidative stress and neurodegenerative diseases: a review of upstream and downstream antioxidant therapeutic options," Current Neuropharmacology, vol. 7, no. 1, pp. 65-74, 2009.

[18] D. Allan Butterfield, B. Howard, S. Yatin et al., "Elevated oxidative stress in models of normal brain aging and Alzheimer's disease," Life Sciences, vol. 65, no. 18-19, pp. 1883-1892, 1999.

[19] M. J. Ball, "Neuronal loss, neurofibrillary tangles and granulovacuolar degeneration in the hippocampus with ageing and dementia. A quantitative study," Acta Neuropathologica, vol. 37, no. 2, pp. 111-118, 1977.

[20] R. H. Swerdlow, “Brain aging, Alzheimer's disease, and mitochondria," Biochimica et Biophysica Acta-Molecular Basis of Disease, vol. 1812, no. 12, pp. 1630-1639, 2011.

[21] J. L. Price, P. B. Davis, J. C. Morris, and D. L. White, “The distribution of tangles, plaques and related immunohistochemical markers in healthy aging and Alzheimer's disease," Neurobiology of Aging, vol. 12, no. 4, pp. 295-312, 1991.
[22] Y.-T. Chang, W.-N. Chang, N.-W. Tsai et al., "The roles of biomarkers of oxidative stress and antioxidant in alzheimer's disease: a systematic review," BioMed Research International, vol. 2014, Article ID 182303, 14 pages, 2014.

[23] M.-C. Badía, E. Giraldo, F. Dasí et al., "Reductive stress in young healthy individuals at risk of Alzheimer disease," Free Radical Biology \& Medicine, vol. 63, pp. 274-279, 2013.

[24] J. K. Andersen, "Oxidative stress in neurodegeneration: cause or consequence?” Nature Medicine, vol. 10, pp. S18-S25, 2004.

[25] L. Ghezzi, "Alzheimer's disease: clinical aspects and treatments," in Neurodegenerative Diseases: Clinical Aspects, Molecular Genetics and Biomarkers, D. Galimberti and E. Scarpini, Eds., chapter 2, pp. 17-26, Springer, London, UK, 2014.

[26] P. A. Adlard, B. A. Tran, D. I. Finkelstein et al., "A review of $\beta$-amyloid neuroimaging in Alzheimer's disease," Frontiers in Neuroscience, vol. 8, article 327, 2014.

[27] W. R. Markesbery, "The role of oxidative stress in Alzheimer disease," Archives of Neurology, vol. 56, no. 12, pp. 1449-1452, 1999.

[28] D. A. Butterfield and C. M. Lauderback, "Lipid peroxidation and protein oxidation in Alzheimer's disease brain: potential causes and consequences involving amyloid $\beta$-peptide-associated free radical oxidative stress," Free Radical Biology and Medicine, vol. 32, no. 11, pp. 1050-1060, 2002.

[29] S. M. Yatin, S. Varadarajan, C. D. Link, and D. A. Butterfield, "In vitro and in vivo oxidative stress associated with Alzheimer's amyloid $\beta$-peptide (1-42)," Neurobiology of Aging, vol. 20, no. 3, pp. 325-330, 1999.

[30] J. Drake, C. D. Link, and D. A. Butterfield, "Oxidative stress precedes fibrillar deposition of Alzheimer's disease amyloid $\beta$ peptide (1-42) in a transgenic Caenorhabditis elegans model," Neurobiology of Aging, vol. 24, no. 3, pp. 415-420, 2003.

[31] D. Praticò, K. Uryu, S. Leight, J. Q. Trojanoswki, and V. M.Y. Lee, "Increased lipid peroxidation precedes amyloid plaque formation in an animal model of Alzheimer amyloidosis," The Journal of Neuroscience, vol. 21, no. 12, pp. 4183-4187, 2001.

[32] S. Hee Lee, I. Kim, and B. Chul Chung, "Increased urinary level of oxidized nucleosides in patients with mild-to-moderate Alzheimer's disease," Clinical Biochemistry, vol. 40, no. 13-14, pp. 936-938, 2007.

[33] M. Y. Aksenov, M. V. Aksenova, D. A. Butterfield, J. W. Geddes, and W. R. Markesbery, "Protein oxidation in the brain in Alzheimer's disease," Neuroscience, vol. 103, no. 2, pp. 373-383, 2001.

[34] B. Su, X. Wang, H.-G. Lee et al., "Chronic oxidative stress causes increased tau phosphorylation in M17 neuroblastoma cells," Neuroscience Letters, vol. 468, no. 3, pp. 267-271, 2010.

[35] S. Tenreiro, K. Eckermann, and T. F. Outeiro, "Protein phosphorylation in neurodegeneration: friend or foe?" Frontiers in Molecular Neuroscience, vol. 7, article 42, 2014.

[36] B. Bhandary, A. Marahatta, H.-R. Kim, and H.-J. Chae, "An involvement of oxidative stress in endoplasmic reticulum stress and its associated diseases," International Journal of Molecular Sciences, vol. 14, no. 1, pp. 434-456, 2013.

[37] N. Gregersen and P. Bross, "Protein misfolding and cellular stress: an overview," Methods in Molecular Biology, vol. 648, pp. $3-23,2010$

[38] F. Shang and A. Taylor, "Ubiquitin-proteasome pathway and cellular responses to oxidative stress," Free Radical Biology \& Medicine, vol. 51, no. 1, pp. 5-16, 2011. 
[39] A. Higa and E. Chevet, "Redox signaling loops in the unfolded protein response," Cellular Signalling, vol. 24, no. 8, pp. 1548$1555,2012$.

[40] M. Padurariu, A. Ciobica, R. Lefter, I. L. Serban, C. Stefanescu, and R. Chirita, "The oxidative stress hypothesis in Alzheimer's disease," Psychiatria Danubina, vol. 25, no. 4, pp. 401-409, 2013.

[41] R. Castellani, K. Hirai, G. Aliev et al., "Role of mitochondrial dysfunction in Alzheimer's disease," Journal of Neuroscience Research, vol. 70, no. 3, pp. 357-360, 2002.

[42] X. Wang, W. Wang, L. Li, G. Perry, H.-G. Lee, and X. Zhu, "Oxidative stress and mitochondrial dysfunction in Alzheimer's disease," Biochimica et Biophysica Acta-Molecular Basis of Disease, vol. 1842, no. 8, pp. 1240-1247, 2014.

[43] T. Malm, M. Mariani, L. J. Donovan, L. Neilson, and G. E. Landreth, "Activation of the nuclear receptor PPAR $\delta$ is neuroprotective in a transgenic mouse model of Alzheimer's disease through inhibition of inflammation," Journal of Neuroinflammation, vol. 12, no. 1, p. 7, 2015.

[44] G. Dentesano, J. Serratosa, J. M. Tusell et al., "CD200R1 and CD200 expression are regulated by PPAR- $\gamma$ in activated glial cells," Glia, vol. 62, no. 6, pp. 982-998, 2014.

[45] D. M. Small, C. Morais, J. S. Coombes, N. C. Bennett, D. W. Johnson, and G. C. Gobe, "Oxidative stress-induced alterations in PPAR- $\gamma$ and associated mitochondrial destabilization contribute to kidney cell apoptosis," American Journal of Physiology-Renal Physiology, vol. 307, no. 7, pp. F814-F822, 2014.

[46] J.-L. Tan, Q.-X. Li, G. D. Ciccotosto et al., "Mild oxidative stress induces redistribution of BACE1 in non-apoptotic conditions and promotes the amyloidogenic processing of Alzheimer's disease amyloid precursor protein," PLoS ONE, vol. 8, no. 4, Article ID e61246, 2013.

[47] S. Miranda, C. Opazo, L. F. Larrondo et al., "The role of oxidative stress in the toxicity induced by amyloid $\beta$-peptide in Alzheimer's disease," Progress in Neurobiology, vol. 62, no. 6, pp. 633-648, 2000.

[48] Y. Christen, "Oxidative stress and Alzheimer disease," The American Journal of Clinical Nutrition, vol. 71, supplement 2, pp. 621S-629S, 2000.

[49] M. A. Deibel, W. D. Ehmann, and W. R. Markesbery, "Copper, iron, and zinc imbalances in severely degenerated brain regions in Alzheimer's disease: possible relation to oxidative stress," Journal of the Neurological Sciences, vol. 143, no. 1-2, pp. 137-142, 1996.

[50] B. S. Berlett and E. R. Stadtman, "Protein oxidation in aging, disease, and oxidative stress," The Journal of Biological Chemistry, vol. 272, no. 33, pp. 20313-20316, 1997.

[51] X. Zhu, A. K. Raina, H.-G. Lee, G. Casadesus, M. A. Smith, and G. Perry, "Oxidative stress signalling in Alzheimer's disease," Brain Research, vol. 1000, no. 1-2, pp. 32-39, 2004.

[52] P. Rinaldi, M. C. Polidori, A. Metastasio et al., "Plasma antioxidants are similarly depleted in mild cognitive impairment and in Alzheimer's disease," Neurobiology of Aging, vol. 24, no. 7, pp. 915-919, 2003.

[53] F. Mangialasche, W. Xu, M. Kivipelto et al., "Tocopherols and tocotrienols plasma levels are associated with cognitive impairment," Neurobiology of Aging, vol. 33, no. 10, pp. 22822290, 2012.

[54] G. C. Román, "Vascular dementia: distinguishing characteristics, treatment, and prevention," Journal of the American Geriatrics Society, vol. 51, supplement 5, pp. S296-S304, 2003.
[55] K. Amar and G. Wilcock, "Vascular dementia," British Medical Journal, vol. 312, no. 7025, pp. 227-231, 1996.

[56] I. Dias, M. Polidori, and H. Griffiths, "Hypercholesterolaemiainduced oxidative stress at the blood-brain barrier," Biochemical Society Transactions, vol. 42, no. 4, pp. 1001-1005, 2014.

[57] F. Derouiche, C. Bôle-Feysot, D. Naïmi, and M. Coëffier, "Hyperhomocysteinemia-induced oxidative stress differentially alters proteasome composition and activities in heart and aorta," Biochemical and Biophysical Research Communications, vol. 452, no. 3, pp. 740-745, 2014.

[58] S. Rubattu, B. Pagliaro, G. Pierelli et al., "Pathogenesis of target organ damage in hypertension: role of mitochondrial oxidative stress," International Journal of Molecular Sciences, vol. 16, no. 1, pp. 823-839, 2015.

[59] F. Di Lisa, N. Kaludercic, A. Carpi, R. Menabò, and M. Giorgio, "Mitochondria and vascular pathology," Pharmacological Reports, vol. 61, no. 1, pp. 123-130, 2009.

[60] H. C. F. Oliveira, R. G. Cosso, L. C. Alberici et al., "Oxidative stress in atherosclerosis-prone mouse is due to low antioxidant capacity of mitochondria," The FASEB Journal, vol. 19, no. 2, pp. 278-280, 2005.

[61] G. Luc and J.-C. Fruchart, "Oxidation of lipoproteins and atherosclerosis," The American Journal of Clinical Nutrition, vol. 53, no. 1, pp. 206S-209S, 1991.

[62] M. C. Polidori, B. Frei, A. Cherubini et al., "Increased plasma levels of lipid hydroperoxides in patients with ischemic stroke," Free Radical Biology \& Medicine, vol. 25, no. 4-5, pp. 561-567, 1998.

[63] K. Gustaw-Rothenberg, K. Kowalczuk, and M. StryjeckaZimmer, "Lipids' peroxidation markers in Alzheimer's disease and vascular dementia," Geriatrics \& Gerontology International, vol. 10, no. 2, pp. 161-166, 2010.

[64] L. Li, R. S. Willets, M. C. Polidori et al., "Oxidative LDL modification is increased in vascular dementia and is inversely associated with cognitive performance," Free Radical Research, vol. 44, no. 3, pp. 241-248, 2010.

[65] L. Nanetti, R. Taffi, A. Vignini et al., "Reactive oxygen species plasmatic levels in ischemic stroke," Molecular and Cellular Biochemistry, vol. 303, no. 1-2, pp. 19-25, 2007.

[66] L. Raz, Q.-G. Zhang, C.-F. Zhou et al., "Role of Racl GTPase in NADPH oxidase activation and cognitive impairment following cerebral ischemia in the rat," PLoS ONE, vol. 5, no. 9, Article ID e12606, 2010.

[67] T. Nagayama, J. Lan, D. C. Henshall et al., "Induction of oxidative DNA damage in the peri-infarct region after permanent focal cerebral ischemia," Journal of Neurochemistry, vol. 75, no. 4, pp. 1716-1728, 2000.

[68] G. A. Rosenberg, N. Sullivan, and M. M. Esiri, "White matter damage is associated with matrix metalloproteinases in vascular dementia," Stroke, vol. 32, no. 5, pp. 1162-1167, 2001.

[69] P. J. Kelly, J. D. Morrow, M. Ning et al., "Oxidative stress and matrix metalloproteinase-9 in acute ischemic stroke: the Biomarker Evaluation for Antioxidant Therapies in Stroke (BEAT-Stroke) Study," Stroke, vol. 39, no. 1, pp. 100-104, 2008.

[70] S. Chrissobolis and F. M. Faraci, "The role of oxidative stress and NADPH oxidase in cerebrovascular disease," Trends in Molecular Medicine, vol. 14, no. 11, pp. 495-502, 2008.

[71] M. L. Modrick, D. A. Kinzenbaw, Y. Chu, C. D. Sigmund, and F. M. Faraci, "Peroxisome proliferator-activated receptor- $\gamma$ protects against vascular aging," American Journal of PhysiologyRegulatory Integrative and Comparative Physiology, vol. 302, no. 10, pp. R1184-R1190, 2012. 
[72] C. Iadecola, "The pathobiology of vascular dementia," Neuron, vol. 80 , no. 4 , pp. 844-866, 2013.

[73] D. Ryglewicz, M. Rodo, P. K. Kunicki et al., "Plasma antioxidant activity and vascular dementia," Journal of the Neurological Sciences, vol. 203-204, pp. 195-197, 2002.

[74] Á. Casado, M. E. López-Fernández, M. C. Casado, and R. de la Torre, "Lipid peroxidation and antioxidant enzyme activities in vascular and Alzheimer dementias," Neurochemical Research, vol. 33, no. 3, pp. 450-458, 2008.

[75] R. N. Kalaria and C. Ballard, "Overlap between pathology of Alzheimer disease and vascular dementia," Alzheimer Disease and Associated Disorders, vol. 13, no. 3, pp. S115-S123, 1999.

[76] S. Bennett, M. M. Grant, and S. Aldred, "Oxidative stress in vascular dementia and alzheimer's disease: a common pathology," Journal of Alzheimer's Disease, vol. 17, no. 2, pp. 245-257, 2009.

[77] B. H. Han, M. L. Zhou, A. W. Johnson et al., "Contribution of reactive oxygen species to cerebral amyloid angiopathy, vasomotor dysfunction, and microhemorrhage in aged Tg2576 mice," Proceedings of the National Academy of Sciences of the United States of America, vol. 112, no. 8, pp. E881-E890, 2015.

[78] G. Paragh, P. Balla, E. Katona, I. Seres, A. Égerházi, and I. Degrell, "Serum paraoxonase activity changes in patients with Alzheimer's disease and vascular dementia," European Archives of Psychiatry and Clinical Neuroscience, vol. 252, no. 2, pp. 63-67, 2002.

[79] R. Esen, M. Aslan, M. E. Kucukoglu et al., "Serum paraoxonase activity, total thiols levels, and oxidative status in patients with acute brucellosis," Wiener Klinische Wochenschrift, 2015.

[80] D. Litvinov, H. Mahini, and M. Garelnabi, "Antioxidant and anti-inflammatory role of paraoxonase 1: implication in arteriosclerosis diseases," North American Journal of Medical Sciences, vol. 4, no. 11, pp. 523-532, 2012.

[81] M. Aviram and M. Rosenblat, "Paraoxonases 1, 2, and 3, oxidative stress, and macrophage foam cell formation during atherosclerosis development," Free Radical Biology \& Medicine, vol. 37, no. 9, pp. 1304-1316, 2004.

[82] D. Commenges, V. Scotet, S. Renaud, H. Jacqmin-Gadda, P. Barberger-Gateau, and J.-F. Dartigues, "Intake of flavonoids and risk of dementia," European Journal of Epidemiology, vol. 16, no. 4, pp. 357-363, 2000.

[83] O. Rozenberg, M. Rosenblat, R. Coleman, D. M. Shih, and M. Aviram, "Paraoxonase (PON1) deficiency is associated with increased macrophage oxidative stress: Studies in PON1knockout mice," Free Radical Biology \& Medicine, vol. 34, no. 6, pp. 774-784, 2003.

[84] L. T. McGrath, B. M. McGleenon, S. Brennan, D. McColl, S. McIlroy, and A. P. Passmore, "Increased oxidative stress in Alzheimer's disease as assessed with 4-hydroxynonenal but not malondialdehyde," QJM, vol. 94, no. 9, pp. 485-490, 2001.

[85] M. Chaves, A. Toral, A. Bisonni et al., "Treatment with vitamin $\mathrm{D}$ and slowing of progression to severe stage of Alzheimer's disease," Vertex, vol. 25, no. 114, pp. 85-91, 2014 (Spanish).

[86] K. H. Masaki, K. G. Losonczy, G. Izmirlian et al., "Association of vitamin $\mathrm{E}$ and $\mathrm{C}$ supplement use with cognitive function and dementia in elderly men," Neurology, vol. 54, no. 6, pp. 12651272, 2000.

[87] C. Zhang, A. Browne, D. Child, and R. E. Tanzi, "Curcumin decreases amyloid- $\beta$ peptide levels by attenuating the maturation of amyloid- $\beta$ precursor protein," The Journal of Biological Chemistry, vol. 285, no. 37, pp. 28472-28480, 2010.
[88] Y. Li, "Protective effects of curcumin on brain vascular dementia by chronic cerebral ischemia in rats and study of the molecular mechanism," Alzheimer's \& Dementia, vol. 7, no. 4, pp. e47-e48, 2011.

[89] A. Clausen, X. Xu, X. Bi, and M. Baudry, "Effects of the superoxide dismutase/catalase mimetic EUK-207 in a mouse model of alzheimer's disease: protection against and interruption of progression of amyloid and tau pathology and cognitive decline," Journal of Alzheimer's Disease, vol. 30, no. 1, pp. 183208, 2012.

[90] X. R. Ma, Z. K. Sun, Y. R. Liu, Y. J. Jia, B. A. Zhang, and J. W. Zhang, "Resveratrol improves cognition and reduces oxidative stress in rats with vascular dementia," Neural Regeneration Research, vol. 8, no. 22, pp. 2050-2059, 2013.

[91] M. J. Barrett, V. Alones, K. X. Wang, L. Phan, and R. H. Swerdlow, "Mitochondria-derived oxidative stress induces a heat shock protein response," Journal of Neuroscience Research, vol. 78, no. 3, pp. 420-429, 2004.

[92] J. F. Abisambra, U. K. Jinwal, J. R. Jones, L. J. Blair, J. Koren III, and C. A. Dickey, "Exploiting the diversity of the heatshock protein family for primary and secondary tauopathy therapeutics," Current Neuropharmacology, vol. 9, no. 4, pp. 623-631, 2011.

[93] J. R. Ou, M. S. Tan, A. M. Xie, J. T. Yu, and L. Tan, "Heat shock protein 90 in Alzheimer's disease," BioMed Research International, vol. 2014, Article ID 796869, 7 pages, 2014.

[94] Y. Chen, B. Wang, D. Liu et al., "Hsp90 chaperone inhibitor 17-AAG Attenuates A $\beta$-induced synaptic toxicity and memory impairment," Journal of Neuroscience, vol. 34, no. 7, pp. 24642470, 2014.

[95] W. Luo, W. Sun, T. Taldone, A. Rodina, and G. Chiosis, "Heat shock protein 90 in neurodegenerative diseases," Molecular Neurodegeneration, vol. 5, no. 1, article 24, 2010.

[96] A. Salminen, J. Ojala, K. Kaarniranta, M. Hiltunen, and H. Soininen, "Hsp90 regulates tau pathology through cochaperone complexes in Alzheimer's disease," Progress in Neurobiology, vol. 93, no. 1, pp. 99-110, 2011.

[97] J. Zou, Y. Guo, T. Guettouche, D. F. Smith, and R. Voellmy, "Repression of heat shock transcription factor HSF1 activation by HSP90 (HSP90 complex) that forms a stress-sensitive complex with HSF1," Cell, vol. 94, no. 4, pp. 471-480, 1998.

[98] N. Fujikake, Y. Nagai, H. A. Popiel, Y. Okamoto, M. Yamaguchi, and T. Toda, "Heat shock transcription factor 1-activating compounds suppress polyglutamine-induced neurodegeneration through induction of multiple molecular chaperones," The Journal of Biological Chemistry, vol. 283, no. 38, pp. 26188-26197, 2008.

[99] N. V. Bobkova, D. G. Garbuz, I. Nesterova et al., "Therapeutic effect of exogenous Hsp70 in mouse models of Alzheimer's disease," Journal of Alzheimer's Disease, vol. 38, no. 2, pp. 425435, 2014.

[100] J. Agulla, D. Brea, F. Campos et al., "In vivo theranostics at the peri-infarct region in cerebral ischemia," Theranostics, vol. 4, no. 1, pp. 90-105, 2014.

[101] G. A. Dienel, M. Kiessling, M. Jacewicz, and W. A. Pulsinelli, "Synthesis of heat shock proteins in rat brain cortex after transient ischemia," Journal of Cerebral Blood Flow and Metabolism, vol. 6, no. 4, pp. 505-510, 1986.

[102] J.-C. L. Plumier, A. M. Krueger, R. W. Currie, D. Kontoyiannis, G. Kollias, and G. N. Pagoulatos, "Transgenic mice expressing 
the human inducible Hsp70 have hippocampal neurons resistant to ischemic injury," Cell Stress \& Chaperones, vol. 2, no. 3, pp. 162-167, 1997.

[103] P. Papadopoulos, P. Rosa-Neto, J. Rochford, and E. Hamel, "Pioglitazone improves reversal learning and exerts mixed cerebrovascular effects in a mouse model of Alzheimer's disease with combined amyloid- $\beta$ and cerebrovascular pathology," PLoS ONE, vol. 8, no. 7, Article ID e68612, 2013.

[104] B. Sharma and N. Singh, "Behavioral and biochemical investigations to explore pharmacological potential of PPAR-gamma agonists in vascular dementia of diabetic rats," Pharmacology Biochemistry and Behavior, vol. 100, no. 2, pp. 320-329, 2011.

[105] R. Ríos-Vázquez, R. Marzoa-Rivas, I. Gil-Ortega, and J. C. Kaski, "Peroxisome proliferator-activated receptor- $\gamma$ agonists for management and prevention of vascular disease in patients with and without diabetes mellitus," American Journal of Cardiovascular Drugs, vol. 6, no. 4, pp. 231-242, 2006.

[106] T. Persson, B. O. Popescu, and A. Cedazo-Minguez, "Oxidative stress in Alzheimer's disease: why did antioxidant therapy fail?" Oxidative Medicine and Cellular Longevity, vol. 2014, Article ID 427318, 11 pages, 2014 


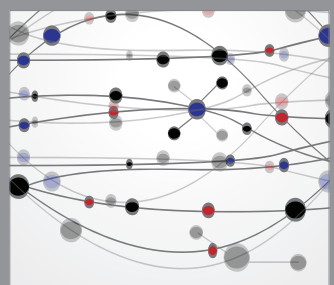

The Scientific World Journal
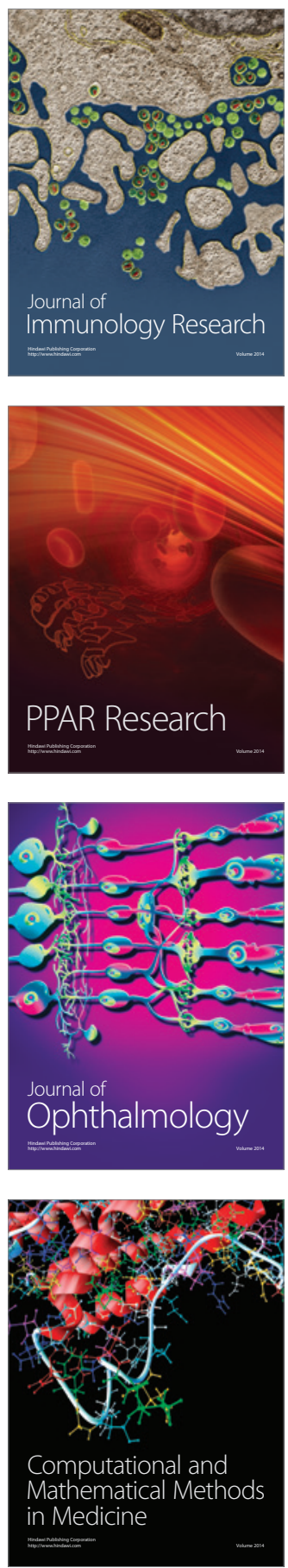

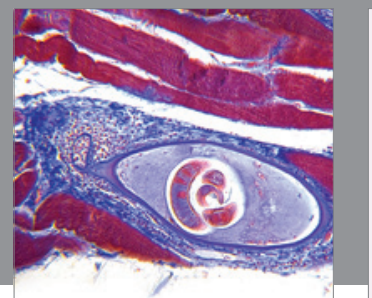

Gastroenterology

Research and Practice
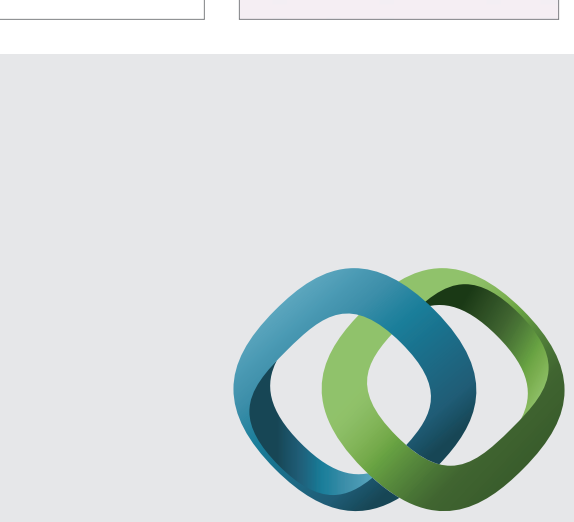

\section{Hindawi}

Submit your manuscripts at

http://www.hindawi.com
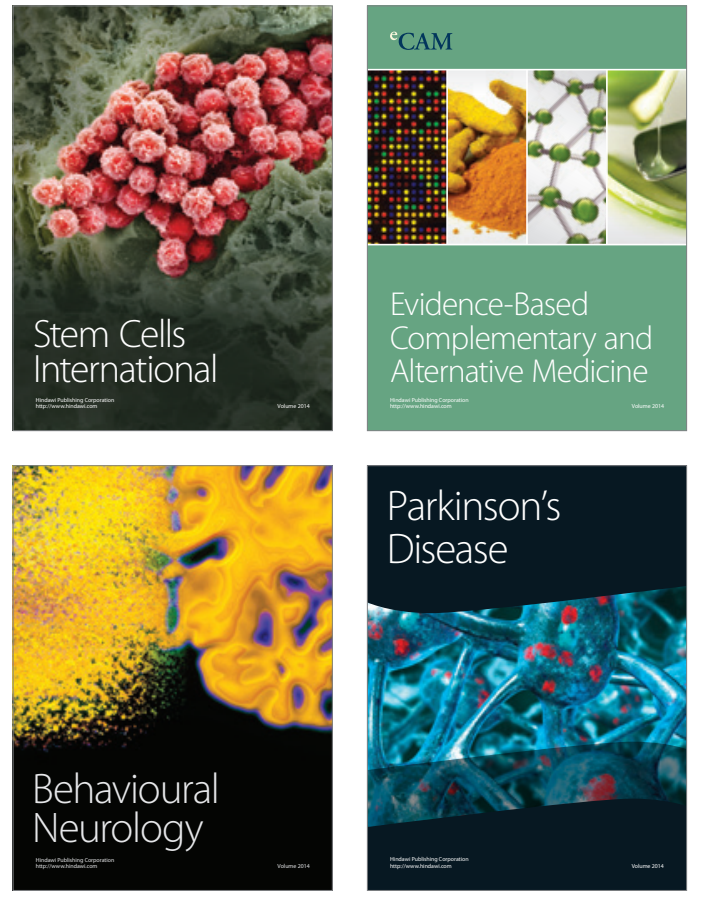
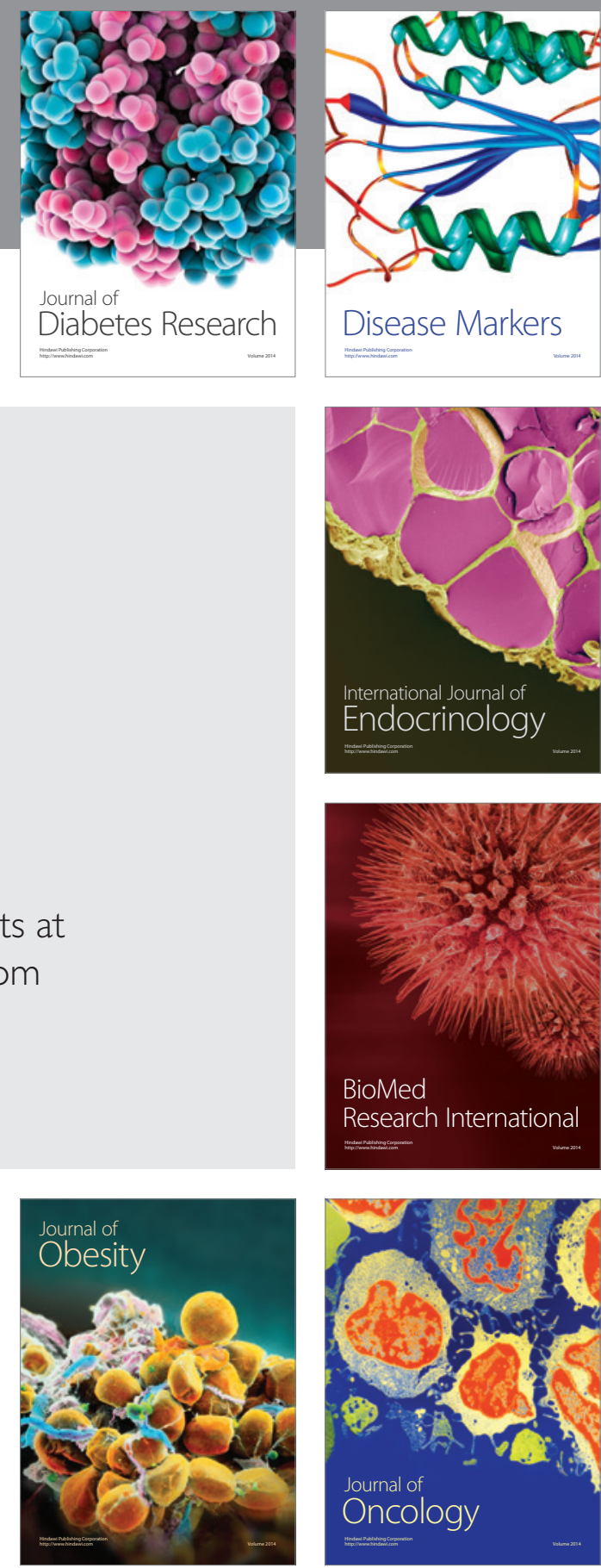

Disease Markers
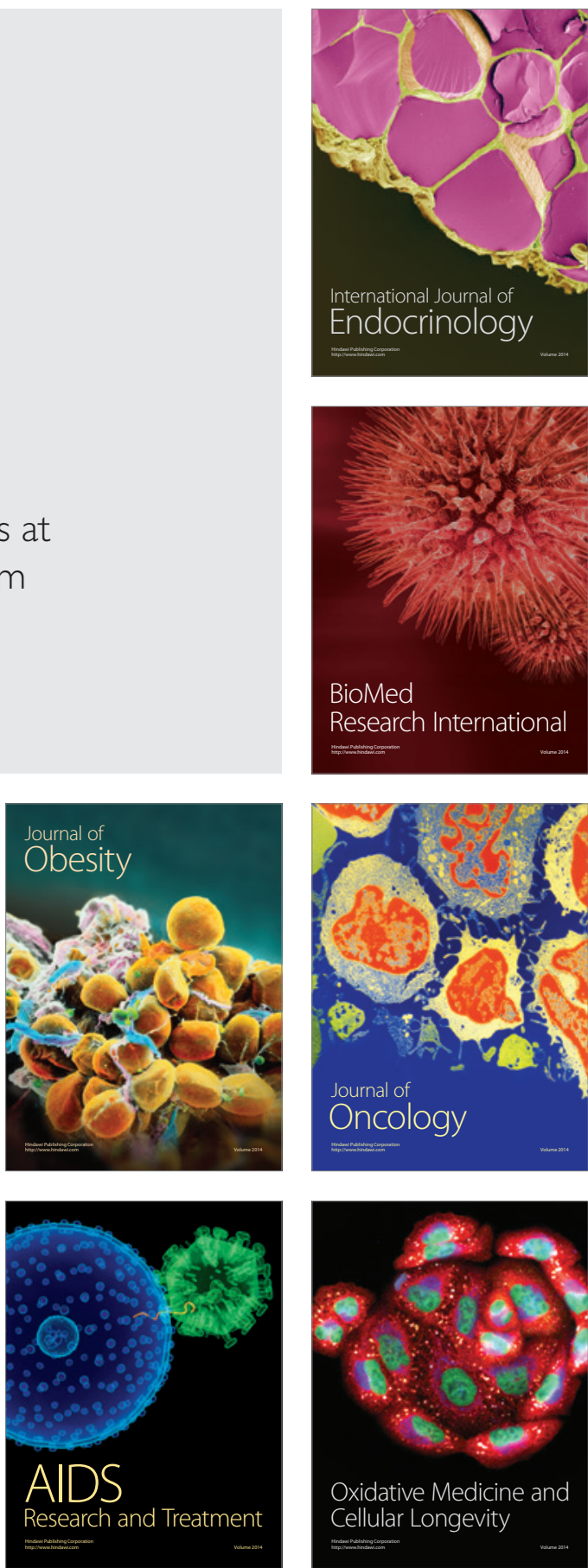